\title{
Considerações Sobre o Escopo de um Processo de Avaliação da Sustentabilidade do Turismo ${ }^{1}$
}

\section{Considerations on the Sustainable Tourism Assessment Process Scoping}

\author{
Itamar Dias e Cordeiro $^{2}$ \\ Maria do Rosário Partidário ${ }^{3}$ \\ Nathália Körössy Leite ${ }^{4}$
}

\begin{abstract}
Resumo
Muito se tem discutido sobre a necessidade do turismo se desenvolver de forma sustentável. Contudo, para que transponha o discurso e se efetive na prática é necessário que existam diretrizes que indiquem quais aspectos devem ser avaliados, pois é somente através da avaliação que há como saber se os resultados esperados foram alcançados. Desta constatação, surge uma pergunta incontornável: o que avaliar? Trata-se de um questionamento que, embora seja fundamental para operacionalizar o conceito de turismo sustentável, tem sido pouco discutido. O presente artigo tem como objetivo sugerir e discutir uma proposta de conteúdo mínimo a ser observada no escopo de qualquer processo direcionado para a avaliação da sustentabilidade do turismo. Como conclusão, sugere-se que para atingir seus propósitos, um processo de avaliação da sustentabilidade no setor do turismo deve: i) adotar uma visão sobre o desenvolvimento sustentável; ii) estabelecer um processo de comunicação efetiva com as partes interessadas; iii) ser capaz de fornecer informações relevantes ao processo de tomada de decisão; iv) contemplar a participação pública; v) ser capaz de se adaptar às novas circunstâncias.
\end{abstract}

Palavras-chave: turismo sustentável; desenvolvimento sustentável; avaliação de sustentabilidade.

\begin{abstract}
A lot has been discussed about the need for tourism to develop in a sustainable way. However, for it to transcend discourse and be made effective into practice, it is necessary the presence of guidelines that indicate which aspects should be evaluated, for it is only through evaluation that one will find if the expected results were achieved. From that finding, arises an unavoidable question: what to evaluate? That questioning is, although fundamental to the operationalization of the sustainable tourism concept, still little discussed. The following article has the goal of suggesting and discussing a minimal content proposal to be observed in the scope of any tourism sustainability evaluation process. As a conclusion, it is suggested that, for it to achieve it's purposes, a tourism sector sustainability evaluation process shall: i)

\footnotetext{
${ }^{1}$ Com o apoio do Programa AlBan, Programa de bolsas de alto nível da União Européia para América Latina.

${ }^{2}$ Turismólogo, Mestre em Ordenamento do Território e Planejamento Ambiental (Universidade Nova de Lisboa); Bolsista do Programa AlBan, bolsa nº E06M103221BR. E-mail: itamar_cordeiro@yahoo.com.br

3 Professora Associada Agregada, DECivil e Arquitectura, Instituto Superior Técnico. E-mail: mrp@civil.ist.utl.pt

${ }^{4}$ Turismóloga, Mestre em Geografia (Universidade de Lisboa); Bolsista do Programa AlBan, bolsa $n^{0}$ E06M103208BR. E-mail: nathaliakorossy@gmail.com
} 
adopt a vision about sustainable development; ii) establish a effective communication process with the stakeholders; iii) be able to provide relevant information to the decision making process; iv) contemplate public participation; v) be able to adapt to new circumstances.

Keywords: sustainable tourism; sustainable development; sustainability assessment.

\section{Introdução}

Conforme enfatizado por Rodrigues (1996), o turismo é incontestavelmente, um fenômeno econômico, político, social e cultural dos mais expressivos das sociedades ditas pósindustrializadas. Nesta perspectiva, a ampla possibilidade que a atividade abre para o desenvolvimento dos países faz com que a mesma seja considerada como um item estratégico, uma vez que pode ajudar a reestruturar territórios que perderam suas vantagens competitivas (Soukiazis \& Proença, 2007).

Não obstante tamanho potencial, verifica-se que ao se desenvolver, o turismo geralmente provoca significativos impactos ambientais, sociais, culturais, econômicos e territoriais (Saarinen, 2006; Hardy \& Beeton, 2001). Assim, face ao reconhecimento de que pode tornarse vítima do seu próprio êxito (Briguglio \& Briguglio, 1996), surge e se afirma a idéia de que a atividade deve se desenvolver de forma sustentável.

De acordo com Butler (apud Sousa, 2006), turismo sustentável é aquele que se desenvolve e se mantém numa área de tal forma que garante a sua viabilidade por um período indefinido de tempo sem degradar ou alterar o ambiente (humano ou físico) e sem pôr em causa o desenvolvimento de outras atividades e processos. Para Swarbrooke (2000), trata-se de um tipo de turismo economicamente viável, mas que não destrói os recursos dos quais a atividade no futuro dependerá, principalmente o ambiente físico e o tecido social da comunidade local. No Acordo de Mohonk ${ }^{5}$ lê-se que um turismo sustentável busca minimizar os impactos ecológicos e sócio-culturais, enquanto promove benefícios econômicos para as comunidades locais e países receptores. Da mesma forma, o que a Carta de Lanzarote ${ }^{6}$ chama de turismo sustentável é um modelo de desenvolvimento turístico ecologicamente suportável em longo

\footnotetext{
${ }^{5} \mathrm{O}$ “Acordo de Mohonk” é um documento que contém um conjunto de princípios gerais e elementos que devem fazer parte de qualquer programa de certificação em ecoturismo e turismo sustentável. Resulta do workshop internacional promovido pelo Institute for Policy Studies com o apoio da Ford Fundation em Mohonk Mountain House (Nova Iorque) em Novembro de 2000. O documento final resultante deste encontro está disponível em «http://www.rainforest-alliance.org/tourism/documents/mohonk.pdf».

6 A “Carta Mundial para o Turismo Sustentável” ou “Carta de Lanzarote” é o documento resultante da Conferencia Mundial do Turismo Sustentável, realizada em Lanzarote (Espanha) em 1995.
} 
prazo, economicamente viável e equitativo do ponto de vista ético e social para as comunidades locais (Fullana \& Ayuso, 2002).

Nesta perspectiva, entende-se, tal como Partidário (2004), que o turismo sustentável não é uma tipologia de turismo (como o são o turismo rural, ecoturismo, turismo de aventuras, etc), mas uma forma diferente de promover turismo. Por isto mesmo, pode ser aplicado a qualquer escala, desde grandes destinos a locais especiais de interesse turístico (Inskeep, 1991). No entanto, não obstante a unanimidade em torno da necessidade de se praticar um turismo sustentável, um dos maiores desafios reside na verificação da real ocorrência deste modelo de desenvolvimento.

Embora a preocupação com a sustentabilidade do turismo já esteja disseminada em muitos governos nacionais, isto, per se, não implica em sua efetiva implementação (Nitsch \& Van der Straaten, 1995). Com efeito, Ruhanem (2004), Simpson (2001) e Trousdale (1999), por exemplo, afirmam que no setor do turismo, de uma forma geral, há um distanciamento crescente entre a “doutrina da sustentabilidade” e sua aplicação ao “mundo real”.

Segundo Ko (2005), a sustentabilidade do turismo tem sido auferida, em grande parte das vezes, com base em julgamentos subjetivos, sem referência a quaisquer padrões ou critérios que suportem as afirmações. Nestas condições, a sustentabilidade no turismo tornou-se, como diriam Saarinen (2006), Garrod \& Fyall (1998) e Hall \& Lew (1998): um jargão para planejadores e políticos, um tema para conferências e um slogan para ativistas ambientais e do desenvolvimento. Para que transponha o discurso e se efetive na prática, é preciso que o turismo sustentável seja avaliado. Desta constatação, surge uma pergunta incontornável: o que avaliar?

O presente artigo tem como objetivo sugerir e discutir uma proposta de conteúdo mínimo a ser observada no escopo de qualquer processo direcionado para a avaliação da sustentabilidade do turismo. Assim, é preciso deixar claro que não se busca discutir a forma mais adequada de avaliação ou como esta deve ser conduzida, e sim o que deve ser avaliado. A discussão é travada, portanto, no plano teórico.

Metodologicamente, a proposta de escopo apresentada neste artigo foi desenvolvida a partir de três conjuntos de ações: i) revisão de literatura; ii) identificação de um modelo de conteúdo de avaliação da sustentabilidade considerado como referência e; iii) adaptação deste modelo às especificidades do turismo. 


\section{Avaliação da Sustentabilidade do Turismo: o quê avaliar?}

Avaliar, segundo Siena (2002, p.76) significa “dar valor”, “julgar”, “determinar a valia, o merecimento”, “ajuizar”. Neste sentido, a avaliação pode ser entendida como o ato de julgar ou determinar o valor, mérito ou qualidade de algo.

As avaliações, em geral, encerram os ciclos de planejamento e constituem uma etapa fundamental, na medida em que servem como um momento de reflexão sobre se o que ocorreu deu-se conforme se esperava. Avaliar é importante porque sem a observação daquilo que foi realizado (ou seja, sem a avaliação) todo o planejamento efetuado perde o sentido, uma vez que não se sabe se os resultados esperados foram alcançados. Logo, mais que uma etapa útil, a avaliação é indispensável à condução de qualquer processo; uma vez que quem não avalia é conduzido por circunstâncias que ignora e somente chegará ao cenário desejado por obra exclusiva do acaso (Barbosa \& Garcia, 2001).

Com o turismo não é diferente (UNWTO, 2004a) e no entender de Beni (2002), a avaliação chega mesmo a ser a fase mais importante de todo o processo de planejamento estratégico do Turismo, já que será com base nos resultados das avaliações que se sustentarão as decisões da Política de Turismo e a estratégia de desenvolvimento do setor. É justamente por ser fortemente marcado pelo discurso e promessa de melhoria que, em turismo, a aferição do que aconteceu constitui uma etapa decisiva.

Para Lee (2006), a avaliação da sustentabilidade deve: considerar aspectos econômicos, ambientais e sociais; prever as condições futuras em diversos cenários; comparar os prováveis efeitos das diferentes ações; e comunicar os resultados de uma forma apropriada às partes interessadas. Para Gasparatos (2008), uma avaliação da sustentabilidade deve: observar, de forma integrada, aspectos econômicos, ambientais, sociais e considerar suas interdependências; considerar as conseqüências das ações presentes no futuro; reconhecer a existência de incertezas com relação ao resultado das ações presentes; envolver o público; e incluir considerações sobre equidade (intra e inter-geracional). Ness et al (2007), por sua vez, consideram que são três os requisitos que devem ser preenchidos numa avaliação da sustentabilidade: capacidade de i) integrar natureza e sociedade; ii) de avaliar diferentes escalas e níveis espaciais; e iii) de avaliar perspectivas de curto e longo prazo.

Contudo, o mais bem estruturado escopo de uma avaliação da sustentabilidade é, provavelmente, aquele formulado por um conjunto de especialistas que se reuniram em 1996, 
em Bellagio (Itália), para discutir o assunto. Para estes especialistas, qualquer processo de avaliação da sustentabilidade deve considerar, pelo menos, 10 princípios básicos (Quadro 1).

\begin{tabular}{l} 
Princípios de Bellagio \\
\hline 1. GUIA DE VISÃO E METAS \\
A avaliação do progresso rumo à sustentabilidade deve: \\
- Ser guiada por uma visão clara do que seja desenvolvimento sustentável e das metas que definam esta \\
visão.
\end{tabular}

\section{PERSPECTIVA HOLÍSTICA}

A avaliação do progresso rumo à sustentabilidade deve:

- Incluir uma revisão do sistema todo e de suas partes;

- Considerar o bem-estar dos subsistemas ecológico, social e econômico, seu estado atual, bem como sua direção e sua taxa de mudança, de seus componentes, e a interação entre as suas partes;

- Considerar as conseqüências positivas e negativas da atividade humana de um modo a refletir os custos e benefícios para os sistemas ecológico e humano, em termos monetários e não-monetários.

\section{ELEMENTOS ESSENCIAIS}

A avaliação do progresso rumo à sustentabilidade deve:

- Considerar a equidade e a disparidade dentro da população atual e entre as gerações presentes e futuras, lidando com a utilização de recursos, superconsumo e pobreza, direitos humanos e acessos a serviços;

- Considerar as condições ecológicas das quais a vida depende;

- Considerar o desenvolvimento econômico e outros aspectos que não são oferecidos pelo mercado e contribuem para o bem-estar social e humano.

\section{ESCOPO ADEQUADO}

A avaliação do progresso rumo à sustentabilidade deve:

- Adotar um horizonte de tempo suficientemente longo para abranger as escalas de tempo humana e dos ecossistemas atendendo às necessidades das futuras gerações, bem como da geração presente em termos de processo de tomada de decisão em curto prazo;

- Definir o espaço de estudo para abranger não apenas impactos locais, mas, também, impactos de longa distância sobre pessoas e ecossistemas;

- Construir um histórico das condições presentes e passadas para antecipar futuras condições.

5. FOCO PRÁTICO

A avaliação do progresso rumo à sustentabilidade deve ser baseada em:

- Um sistema organizado que relacione as visões e metas dos indicadores e os critérios de avaliação;

- Um número limitado de questões-chave para análise;

- Um número limitado de indicadores ou combinação de indicadores para fornecer um sinal claro do progresso;

- Na padronização das medidas quando possível para permitir comparações;

- Na comparação dos valores dos indicadores com as metas, valores de referência, padrão mínimo e tendências.

6. ABERTURA/TRANSPARÊNCIA

A avaliação do progresso rumo à sustentabilidade deve:

- Construir os dados e indicadores de modo que sejam acessíveis ao público;

- Tornar explícitos todos os julgamentos, suposições e incertezas nos dados e nas interpretações.

7. COMUNICAÇÃO EFETIVA

A avaliação do progresso rumo à sustentabilidade deve:

- Ser projetada para atender às necessidades do público e do grupo de usuários;

- Ser feita de uma forma que os indicadores e as ferramentas estimulem e engajem os tomadores de decisão;

- Procurar a simplicidade na estrutura do sistema e utilizar linguagem clara e simples.

8. AMPLA PARTICIPAÇÃO

A avaliação do progresso rumo à sustentabilidade deve:

- Obter ampla representação do público: profissional, técnico e comunitário, incluindo participação de jovens, mulheres e indígenas para garantir o reconhecimento dos valores, que são diversos e dinâmicos;

- Garantir a participação dos tomadores de decisão para assegurar uma forte ligação na adoção de políticas e nos resultados da ação. 


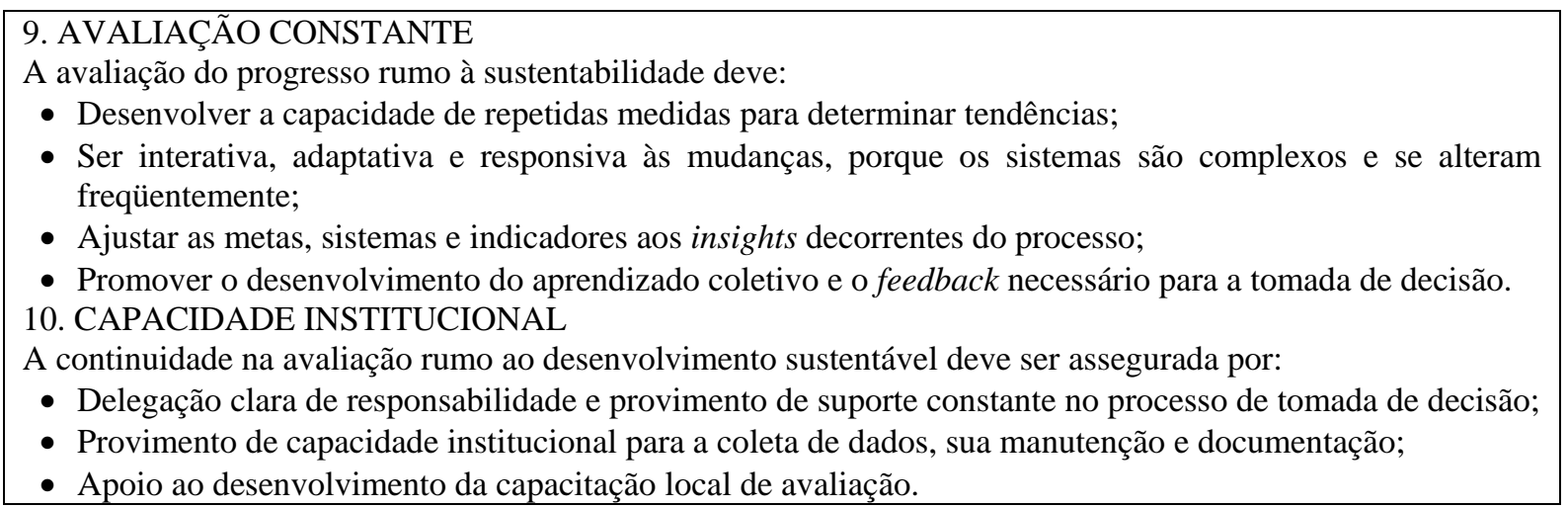

Quadro 1 - Princípios de Bellagio

Fonte: Hardi \& Zdan (1997)

Em síntese, o que estes princípios representam é exatamente aquilo que se espera de um exercício de avaliação de sustentabilidade: geração de informações úteis e confiáveis acerca do estágio de desenvolvimento de determinada realidade, identificando problemas e limitações, potencialidades e alternativas de modo que as parte interessadas na atividade possam ter uma imagem adequada do rumo que está sendo seguido.

Os Princípios de Bellagio não são orientados especificamente para o turismo; no entanto, segundo Ko (2005), podem ser perfeitamente aplicáveis ao processo de avaliação da sustentabilidade da atividade. Assim, uma vez que sintetizam a percepção geral sobre os aspectos relacionados com a avaliação do desenvolvimento sustentável e considerando que inexiste uma carta de princípios semelhante específica para o turismo, fez-se uso dos Princípios de Bellagio como marco de referência para delimitar o escopo do que deve ser observado num processo de avaliação da sustentabilidade do turismo.

\section{Aspectos Essenciais a Serem Observados Numa Avaliação da Sustentabilidade no}

\section{Turismo}

Nos Princípios de Bellagio, o princípio 1 recomenda que a avaliação do progresso rumo à sustentabilidade deve ser guiada por uma visão clara do que seja desenvolvimento sustentável e das metas que definam esta visão. Os princípios 2 ao 5 sintetizam a necessidade de uma abordagem holística (o que requer necessariamente a preocupação com os subsistemas ecológico, social e econômico, bem como a interação entre eles) e que tenha em observância a questão da justiça intergeracional. Os princípios 6 e 7 reclamam a necessidade de uma 
avaliação transparente (openness), cujos resultados sejam inteligíveis pelo público e no qual todos os julgamentos, suposições e incertezas nos dados e nas interpretações sejam abertamente colocados. Além disso, a avaliação rumo à sustentabilidade deve prezar pela simplicidade e clareza na estrutura do sistema e utilizar linguagem clara e simples em sua comunicação ao público. O princípio 8 ressalta a necessidade de haver uma ampla participação como forma de legitimação do processo. O princípio 9 enfatiza a necessidade de que a avaliação seja constante, adaptativa e responsiva às mudanças, afinal a realidade é complexa e se altera freqüentemente; um instrumento de avaliação incapaz de se adaptar a estas mudanças não tem como cumprir com suas funções. Finalmente, o princípio 10 refere-se àquilo que mais se espera de um instrumento de avaliação: suporte ao processo de tomada de decisão, pois, em última instância, avaliar serve justamente para obter as informações necessárias para seguir rumo ao fim desejado.

De forma estrutural e observando o caso específico do turismo, estes 10 princípios foram sintetizados e agrupados em 5 categorias que, entende-se, devem ser observadas em qualquer processo de avaliação da sustentabilidade do turismo. São elas: i) visão de desenvolvimento sustentável; ii) comunicação efetiva; iii) capacidade de apoio à decisão; iv) participação pública; v) potencial para avaliação constante. Discute-se a seguir, em pormenor, o que deve ser avaliado dentro de cada uma dessas categorias.

\subsection{Visão de desenvolvimento sustentável}

O primeiro passo num processo de avaliação da sustentabilidade é a definição do conceito/visão de desenvolvimento sustentável (Pope et al, 2004). Isto porque o sucesso de qualquer processo de avaliação está fortemente vinculado a uma definição clara do objeto em análise, uma vez que quanto mais claros e definidos estiverem os conceitos, maior precisão (ou menor chances de distorções) se conseguirá (Mousinho, 2001). Se houver qualquer tipo de dúvida, equívoco terminológico ou conceitos implícitos sobre aquilo que se quer avaliar, o alicerce do próprio processo de avaliação torna-se frágil. Se isto acontecer, a avaliação fica sujeita a viesses e abrem-se precedentes para contestações sobre os resultados.

Assim, considerando que uma definição clara é necessária para evitar confusões ou equívocos (Hardi \& Barg, 1997), algo muito comum neste campo de investigação (Ko, 2005), adota-se 
neste trabalho a definição de sustentabilidade mundialmente mais conhecida e aceita: a do Relatório Brundtland ${ }^{7}$.

Adotar tal conceito é, em última análise, acatar duas premissas: i) a de que o desenvolvimento deve pesar equitativamente o crescimento econômico, a justiça social e a proteção do ambiente; e ii) observar a questão da equidade inter e intra-geracional. Medir e avaliar proteção ao meio ambiente, desenvolvimento social e crescimento econômico requer que estas dimensões sejam consideradas de forma igual, sem que uma se sobreponha à outra, como, aliás, é corriqueiro quando se analisa a sustentabilidade (Ko, 2005). A questão da equidade inter/intra-geracional é ainda mais complicada. Embora esta seja uma das características mais marcantes do processo de desenvolvimento sustentável, segundo a definição do Relatório Brundtland, não há, atualmente, uma forma sistematizada de analisar tal aspecto, de modo que se trata, por enquanto, de um aspecto mais teórico do que prático.

\subsection{Comunicação efetiva}

Se é verdade que o progresso rumo ao desenvolvimento sustentável é uma questão de escolha (Hardi \& Zdan, 1997; Parris \& Kates, 2003) e que a mudança só é possível com a participação pública (Hardi \& Barg, 1997), é evidente que a população somente poderá optar pelo desenvolvimento sustentável e participar neste modelo de desenvolvimento na medida em que se encontrar devidamente informada. No entanto, como destaca Guerreiro (2004, p.81), vivese hoje num contra-senso, pois embora haja "um dilúvio de informações”, a população encontra-se “totalmente desinformada”. O desafio, portanto, é encontrar formas de organização da informação e fazê-las chegarem às pessoas.

Transmitir de forma eficiente, uma mensagem confusa ou indecifrável, em nada colabora com o processo de desenvolvimento sustentável, pelo contrário, pode mesmo levar ao desinteresse da população (Hammond et al, 1995). Infere-se, daí, que comunicar não se resume a transmitir a mensagem ao público, mas é também fazê-lo de maneira inteligível (Fullana \& Ayuso, 2001). No entanto, em grande parte, o que se verifica é que os vários métodos de avaliação da sustentabilidade geram resultados que não são facilmente inteligíveis ou comunicáveis à comunidade ou aos tomadores de decisões (Graymore, 2005) tendo, em sua maioria, seu entendimento restrito a especialistas da área.

\footnotetext{
${ }^{7}$ A publicação do Relatório Brundtland, se não dirimiu, pelo menos minimizou a confusão sobre o termo “desenvolvimento sustentável”. Forneceu uma baliza internacional sem dúvida mais precisa que as tentativas precursoras (Veiga, 2005, p.196).
} 
Concomitantemente, deve-se atentar para o fato de que análises neutras com interpretações estritamente técnicas não passam de mitos (Vicente \& Partidário, 2006). Não existe método de avaliação asséptico e desprovido de concepções filosóficas e/ou ideológicas (Siena, 2002), uma vez que a avaliação envolve valores e julgamentos sobre o modelo a ser usado e a meta a atingir (Parris \& Kates, 2003), seja na coleta de dados e informações, seja na fase de interpretação. Ademais, há sempre o risco de manipulação da informação por razões políticas, considerações emocionais ou conservacionistas ou por pressão de determinados grupos (Somerville apud Mousinho, 2001).

Logo, para gozar da legitimidade necessária que este tipo de ação requer, é necessário que o processo de avaliação de sustentabilidade comunique abertamente quais são seus valores, pressupostos, julgamentos, métodos e incertezas (Partidário, 2007; Hammond et al, 1995). O propósito é que quem está a ser informado saiba como se chegou aquele resultado e as pessoas em desacordo possam ver como julgamentos alternativos alterariam a avaliação (Siena, 2002). É o que nos Princípios de Bellagio se denomina de abertura/transparência (openness) e que, segundo Hardi \& Barg (1997), é componente obrigatória em uma comunicação efetiva da sustentabilidade.

Assim, no que respeita à capacidade de comunicação, considera-se que a avaliação da sustentabilidade do turismo deve ser transparente e possuir um bom nível de apresentação. Por transparência, entenda-se o grau de facilidade para compreender o método de avaliação e os julgamentos de valor empregados; enquanto a apresentação ou interface (van Bellen, 2005; Delai \& Takahashi, 2007), refere-se à clareza da mensagem e ao modo como os resultados são visualmente transmitidos.

\subsection{Capacidade de apoio a decisão}

Como lembra Mousinho (2001), os usuários de uma avaliação são dois: o público e os tomadores de decisões ${ }^{8}$. Assim, torna-se igualmente relevante, para além de refletir sobre a relação dos instrumentos de avaliação com o público, verificar como estes instrumentos facilitam a informação a um grupo específico de atores: os tomadores de decisões (UNWTO, 2004a; Hammond et al, 1995). Isto porque a este grupo, cabe analisar a realidade e, face às

\footnotetext{
${ }^{8}$ Tomada de decisão, segundo UNCSD (apud Mousinho, 2001, p.09), é um processo cíclico, que em geral envolve cinco etapas, havendo necessidade de informação disponível para cada uma delas: identificação do problema; formulação de políticas; implementação; monitoramento do desempenho e avaliação.
} 
alternativas existentes, optar por aquelas que melhores chances terão em conduzir o turismo rumo à sustentabilidade.

Ora, partindo-se do argumento de que as pessoas não podem responder com informações que não têm e não podem reagir com informações inadequadas (Meadows, 1998); não há como seguir rumo ao desenvolvimento sustentável sem informações. Com efeito, gerir um destino, sobretudo de forma sustentável, requer informações. Assim, entre outras coisas, um instrumento de avaliação de sustentabilidade precisa estar apto a facultar aos gestores o que estes precisam saber para reduzirem os riscos de tomarem decisões equivocadas (UNWTO, 2004a). Mas no caso do turismo, que tipo de informações os decisores precisam que os sistemas de avaliação lhes forneçam? Em outras palavras: o que os tomadores de decisões, efetivamente, precisam saber para gerir o destino de forma sustentável?

Segundo UNWTO (2004a), o que fundamentalmente interessa aos decisores são as informações que permitam detectar riscos, o que é diferente de detectar impactos negativos 9 . No entanto, o fato de serem direcionadas ao mais alto nível de decisão não implica que as informações devam ser complexas ou extremamente elaboradas. Pelo contrário, embora tratem de um fenômeno complexo, os métodos que procuram avaliar a sustentabilidade devem prezar pela simplicidade da informação gerada (van Bellen, 2005).

Em geral, os tomadores de decisões não dispõem de tempo para analisar informações complexas. Por isso mesmo, necessitam que estas sejam simples e representativas. Precisam de informações com as quais possam traçar um quadro preciso das condições existentes num dado momento para, a partir daí, dedicarem-se à formulação de estratégias. No entanto, o que se verifica na prática é algo diferente.

CCE (2003), identifica que no campo do turismo sustentável, são dois os principais obstáculos à transposição de iniciativas para ações concretas bem sucedidas: i) o fato dos gestores serem confrontados com muitas questões e ii) o fato de haver uma comunicação inadequada baseada em mensagens formuladas numa linguagem complexa que não chegam aos intervenientes ao nível local e regional, nem aos cidadãos.

\footnotetext{
${ }^{9}$ Segundo Partidário (2007), oportunidades e riscos correspondem, respectivamente, aos impactos positivos e negativos de natureza estratégica. As oportunidades (impactos estratégicos positivos) e os riscos (impactos estratégicos negativos) constituem a avaliação da forma como se prevê que os valores ambientais, sociais e culturais venham a ser utilizados e a sua integridade afetada, e o que isso pode significar sobre os processos de desenvolvimento sustentável.
} 
Disto, infere-se que, para ser útil à tomada de decisões, um processo de avaliação da sustentabilidade do turismo deve ser formulado com a preocupação de gerar informações simples e significativas. Enquanto simplicidade refere-se à clareza e objetividade da informação gerada; significância tem a ver com a capacidade da informação gerada ser relevante e expressiva para o planejamento, nomeadamente no sentido de alertar para riscos que possam comprometer os objetivos de sustentabilidade do turismo.

\subsection{Participação pública}

Outro aspecto significativo numa avaliação da sustentabilidade é a forma e a intensidade através das quais diferentes atores sociais participam do processo. Esta característica constitui, segundo os Princípios de Bellagio, um importante elemento que deve estar presente em qualquer tentativa de avaliação. Da mesma forma, a Agenda 21, em seu capítulo 23, deixa bem claro que um dos pré-requisitos fundamentais para a realização do desenvolvimento sustentável é a ampla participação pública. As Declarações de Lanzarote, de Berlim e de Hainan, em seus respectivos “Princípio 4”, "Preâmbulo” e "Princípio 2”, enfatizam o mesmo para o turismo sustentável.

Participação pública diz respeito ao envolvimento, informação e consulta das partes interessadas em atividades de planejamento, gestão ou outras atividades em que haja lugar ações voltadas para tomar decisões a respeito de determinado assunto (Clark, 1999). Parte interessada (stakeholder), por sua vez, é qualquer pessoa, grupo ou organização que pode ser, ou é, afetado pela realização de determinada ação (Freeman apud Sautter \& Leisen, 1999).

De acordo com Clark (1999), a pressão para que o público tenha um papel cada vez mais importante no processo de planejamento e avaliação, fundamenta-se em considerações filosóficas e pragmáticas. As primeiras estão principalmente relacionadas com a crença generalizada de que em muitas sociedades um indivíduo tem o direito de se envolver, ser informado, consultado e de exprimir seus pontos de vista sobre matérias que o afetam pessoalmente. As considerações de natureza pragmática dizem respeito à incapacidade das decisões tomadas por terceiros identificarem corretamente as preferências do público.

Por estas razões, a participação constitui uma importante dimensão de qualquer processo de avaliação, motivo pelo qual deve ser séria e amplamente considerada (van Bellen, 2005; Bossel, 1999; Clark, 1999; Meadows, 1998). Por outro lado, é prudente considerar que, embora a ausência de participação enfraqueça substancialmente a credibilidade e a 
legitimidade do processo de avaliação, não se pode afirmar que necessariamente o nulifica ou invalida (UNESCO/SCOPE, 2006).

Há, correntemente, duas formas de se trabalhar com participação pública em processos de avaliação da sustentabilidade: do topo para baixo (top-down) e da base para cima (bottom-up). De acordo com van Bellen (2005), na abordagem top-down, o processo é coordenado por especialistas que definem o método de avaliação e o grau de participação pública que o mesmo terá. A vantagem desta abordagem é que fornece uma aproximação cientificamente mais homogênea e mais válida. A desvantagem é que não há um contato direto com as prioridades das comunidades. Na abordagem bottom-up, o processo é bem mais participativo e é conduzido pelos diversos atores sociais envolvidos (como líderes, tomadores de decisão, comunidade). A principal vantagem é que a comunidade realmente adota o projeto. A principal limitação é o foco estreito que pode levar à omissão de aspectos essenciais à sustentabilidade.

Imperativo referenciar que são abordagens diferentes, perspectivas distintas de como trabalhar com a participação pública. Nenhuma delas é necessariamente certa ou errada, de modo que não cabe aqui fazer um julgamento de valor a este respeito. O que se quer chamar a atenção é que um processo de avaliação da sustentabilidade do turismo deve ter bastante claro a existência destas duas abordagens a adotar aquela que considerar mais conveniente, justificando o motivo da escolha.

\subsection{Potencial para avaliação constante}

Por fim, cumpre ter claro que a sustentabilidade também envolve uma dimensão temporal (Bossel, 1999). Um cenário pode ser sustentável hoje e pode não o ser no futuro. A realidade é um sistema complexo e sistemas complexos mudam constantemente (McKercher, 1999). É sobre este comportamento dinâmico que se espera que o processo de avaliação esteja preparado para contemplar.

O turismo é um fenômeno complexo e dinâmico (Farrell \& Twining-Ward, 2005; Beni, 2002; McKercher, 1999), assim como o próprio desenvolvimento sustentável (Bossel, 1999). Logo, existe uma necessidade de que seja gerido consoante as (novas) circunstâncias que freqüentemente se apresentam (Yuksel et al, 1999), e neste contexto, modelos de avaliação estáticos têm pouca ou nenhuma serventia. Tal característica irá demandar a capacidade de captar as alterações, alertando, quando for o caso, de se estar afastando do cenário desejado. 
Trata-se, em última análise, de uma capacidade para avaliar de forma contínua; que, aliás, nada mais é que uma característica intrínseca à própria lógica e natureza do processo de avaliação.

Ao mesmo tempo, um adequado processo de avaliação deve estar preparado para diagnosticar tendências. Determinar tendências significa, em última instância, captar a situação de um determinado destino turístico ao longo de várias “janelas” no tempo, de modo a perceber se o mesmo inclina-se para o sustentável ou insustentável. Está, portanto, relacionado com a capacidade de antecipar o que irá ocorrer com base no que se vem analisando. Não se trata, portanto, de uma capacidade de prever o futuro, mas de especular o que provavelmente irá ocorrer com base no que aconteceu e no que vem sendo observado.

Em suma, qualquer processo de avaliação da sustentabilidade deve ser concebido numa perspectiva de avaliação constante, o que significa que deve possuir a capacidade de se adaptar às circunstâncias dinâmicas que envolvem o desenvolvimento sustentável, incorporando novas variáveis que se mostrem relevantes ao longo do tempo. Além disso, deve se preocupar com a previsão de tendências, acompanhando a evolução dos acontecimentos e, com base nisso, estabelecer possíveis desdobramentos futuros.

\section{Considerações Finais}

Colocado como o principal desafio da humanidade ao longo do século XXI, o desenvolvimento sustentável configura-se cada vez mais como um fim a ser alcançado pelas várias atividades econômicas. Com o turismo, uma das principais atividades econômicas em nível mundial, não é diferente. No entanto, desenvolvimento sustentável e turismo sustentável são apenas conceitos e como em todo conceito, só há uma forma de constatar sua operacionalização: através da avaliação.

Por outro lado, há que se reconhecer que avaliar algo como o turismo sustentável não é algo simples. Questões relacionadas a "o quê avaliar" constituem uma das primeiras e principais dificuldades, uma vez que sem saber o quê efetivamente avaliar, dificilmente passar-se-á do discurso para a prática. Neste sentido, o presente trabalho apresentou algumas considerações sobre qual o conteúdo que deve estar presente numa avaliação da sustentabilidade do turismo. 
Reconhecendo-se que se trata muito mais de um convite à discussão do que uma palavra final sobre o assunto, entende-se que as idéias aqui discutidas fornecem uma colaboração útil na medida em que as discussões sobre o assunto ainda são incipientes. Considera-se que as iniciativas de avaliação da sustentabilidade do turismo que adotem uma visão sobre o desenvolvimento sustentável; estabeleçam um processo de comunicação efetiva com as partes interessadas; sejam capazes de fornecer informações relevantes ao processo de tomada de decisão; possibilitem a participação pública e; sejam capazes de se adaptar às novas circunstâncias, tenderão a lograr êxito.

\section{Referências}

BARBOSA, Frederico; GARCIA, Ronaldo. 2001. A propósito da avaliação do PPA: Lições da primeira tentativa. Boletim de Políticas Sociais: Acompanhamento e Análise, $\mathrm{n}^{\circ}$ 3, pp.121-125. IPEA. Disponível em: <http://www.ipea.gov.br/sites/000/2/publicacoes/bpsociais/bps_03/ensaio2_proposito. pdf>. Acesso em: 16 dez. 2007.

BENI, Mário. 2002. Análise estrutural do turismo. 7. ed. São Paulo: SENAC.

BOSSEL, Hartmut. 1999. Indicators for sustainable development: Theory, method, applications. Winnipeg. Disponível em: <http://www.iisd.org/pdf/balatonreport.pdf>. Acesso em: 17 out. 2007.

BRIGUGLIO, L.; BRIGUGLIO, M. 1996. Sustainable tourism in the Maltese Islands. In: BRIGUGLIO, L.; BUTLER, R; HARRISON, D; LEAL FILHO, W. (ed.). Sustainable tourism in islands \& small states: Case studies. Pinter: London. p. 162-179

CCE - COMISSÃO DAS COMUNIDADES EUROPEIAS. 2003. Orientações de base para a sustentabilidade do turismo europeu. Bruxelas, 21.11.2003, COM (2003) 716 Final.

CLARK, B. 1999. Participação pública. In. PARTIDÁRIO, M.R.; JESUS, J. (eds). Avaliação do impacte ambiental: Conceitos, procedimentos e aplicações. Centro de Estudos de Planeamento e Gestão do Ambiente: Caparica, p. 165-171.

DELAI, Ivete; TAKAHASHI, Sérgio. 2007. Uma proposta de modelo de referencia para mensuração da sustentabilidade corporativa. In: Anais do IX Encontro Nacional sobre gestão empresarial e meio ambiente. ENGEMA: Curitiba. Disponível em: <http://engema.unicenp.edu.br/arquivos/engema/ pdf/PAP0255.pdf $>$. Acesso em: 10 fev. 08.

FARRELL, Bryan.; TWINING-WARD, Louise. 2005. Seven steps towards sustainability: Tourism in the context of new knowledge. Journal of Sustainable Tourism, v.13, n.2, p.109-122.

FULLANA, Pere; AYUSO, Silvia. 2002. Turismo sostenible. Rubes Editorial: Barcelona.

GARROD, Brian; FYALL, Allan. 1998. Beyond the rethoric of sustainable tourism? Tourism Management, v.19 n.3, p.199-212.

GASPARATOS, Alexandros; EL-HARAM, Mohamed; HORNER, Malcolm. 2008. A critical review of reductionist approaches for assessing the progress towards sustainability. Environmental Impact Assessment Review, v.28, n.4-5, p.286-311.

GRAYMORE, Michelle. 2005. Journey to sustainability: Small regions, sustainable carrying capacity and sustainability assessment methods. Tese (Doutorado) - Griffith University, Queensland. 
GUERREIRO, Cristina. 2004. Indicadores de desenvolvimento sustentável, aplicados em sistemas de informação geográfica (SIG), para o litoral norte da Bahia. 2004. Dissertação (Mestrado em Desenvolvimento Sustentável) - Universidade de Brasília, Brasília.

HALL, Michael; LEW, Alan. 1998. The geography of sustainable tourism: Lessons and prospects. In: HALL, Michael \& LEW, Alan (eds.). Sustainable tourism: A geographical perspective. Longman, New York, p. 199-203.

HAMMOND, Allen; ADRIANNSE, Albert; RODENBURG, Eric; BRYANT, Dirk; WOODWARD, Richard. 1995. Environmental indicators: A systematic approach to measuring and reporting on environmental policy performance in the context of sustainable development. World Resources Institute: Washington, DC.

HARDI, Peter, BARG, Stephan. 1997. Measuring sustainable development: Review of current practice. IISD: Winnipeg. Winnipeg.

; ZDAN, Terrence. 1997. Assessing sustainable development: Principles in practice. IISD:

HARDY, A.; BEETON, R. 2001. Sustainable tourism or maintainable tourism: Managing resources for more than average outcomes. Journal of Sustainable Tourism, v.9, n.3, p.168-192.

INSKEEP, Edward. 1991. Tourism planning: An integrated and sustainable development approach. Van Nostrand Reinhold: New York.

KO, Tae Gyou. 2005. Development of a tourism sustainability assessment procedure: a conceptual approach. Tourism Management, v.26, n.3, p.431-445.

KRIPPENDORF, Jost. 2003. Sociologia do turismo: Para uma nova compreensão do lazer e das viagens. $3^{\mathrm{a} e d .}$ Aleph: São Paulo.

LEE, Norman. 2006. Bridging the gap between theory and practice in integrated assessment. Environmental Impact Assessment Review, v.26, n.1, p.57-78.

MCKERCHER, Bob. 1999. A chaos approach to tourism. Tourism Management, v.20, n.4, p.425434.

MEADOWS, Donella. 1998. Indicators and information systems for sustainable development. The Sustainability Institute: Hartland. Disponível em: <http://www.iisd.org/pdf/s_ind_2.pdf > . Acesso em: 10 out. 07.

MOUSINHO, Patrícia. 2001. Indicadores de desenvolvimento sustentável: Modelos internacionais e especificidades do Brasil. Dissertação (Mestrado em Ciência da Informação) - Universidade Federal do Rio de Janeiro, Rio de Janeiro.

NESS, Barry; URBEL-PIIRSALU, Evelin; ANDERBERG, Stefan; OLSSON, Lennart. 2007. Categorising tools for sustainability assessment. Ecological Economics, v.60, n.3, p.498-908.

NITSCH, Brigitte; VAN DER STRAATEN, Jan. 1995. Rural tourism development: Using a sustainable tourism development approach. In: COCCOSSIS, Harry; NIJKAMP, Peter (eds.). Sustainable Tourism Development: Ashgate, p.169-185.

PARRIS, Thomas; KATES, Robert. 2003. Characterizing and measuring sustainable development. Annual Review of Environment and Resources, v.28, n.1, p.559-586.

PARTIDÁRIO, Maria Rosário. 2007. Guia de boas práticas para Avaliação Ambiental Estratégica: Orientações metodológicas. Agência Portuguesa do Ambiente: Lisboa.

2004. Integração do conceito de desenvolvimento sustentável no turismo nacional. Jornal Água\&Ambiente, v.69, p.26-27. 
POPE, Jenny; ANNANDALE, David; MORRISON-SAUNDERS, Angus. 2004. Conceptualising sustainability assessment. Environmental Impact Assessment Review. v.24, n.6, p.595-616.

RODRIGUES, Adyr. 1996. Desafios para os estudiosos do turismo. In: RODRIGUES, Adyr. Turismo e geografia: Reflexões teóricas e enfoques regionais. Hucitec: São Paulo, p.17-32.

RUHANEM, Lisa. 2004. Strategic planning for local tourism destinations: An analysis of tourism plans. Tourism and Hospitality Planning \& Development, v.1, n.3, p.239-253.

SAARINEN, Jarkko. 2006. Traditions of sustainability in tourism studies. Annals of Tourism Research, v.33, p.4, p.1121-1140.

SAUTTER, Elise; LEISEN, Brigit. 1996. Managing stakeholders: A tourism planning model. Annals of Tourism Research, v.26, n.2, pp.312-328.

SIENA, Osmar. 2002. Método para avaliar o progresso em direção ao desenvolvimento sustentável. Tese (Doutorado em Engenharia de Produção) - Universidade Federal de Santa Catarina, Florianópolis.

SIMPSON, Ken. 2001. Strategic planning and community involvement as contributors to sustainable tourism development. Current Issues in Tourism, v.4, n.1, pp.03-41.

SOUKIAZIS, Elias; PROENÇA, Sara. 2007. Tourism as an alternative source of regional growth in Portugal: A panel data analysis at NUTS II and III levels. Portuguese Economic Journal, v.7, n.1, pp.43-61.

SOUSA, Renata. 2006. A sustentabilidade do destino turístico Porto Santo. Dissertação (Mestrado em Gestão Estratégica e Desenvolvimento do Turismo) - Universidade da Madeira, Porto Santo.

SWARBROOKE, John. 2000. Turismo sustentável. São Paulo: Aleph.

TROUSDALE, William. 1999. Governance in context, Boracay Island, Philippines. Annals of Tourism Research, v.26, n.4, pp.840- 867.

UNESCO/SCOPE - UNITED NATIONS EDUCATIONAL, SCIENTIFIC AND CULTURAL ORGANIZATION/ SCIENTIFIC COMMITTEE ON PROBLEMS OF THE ENVIRONMENT OF INTERNATIONAL COUNCIL FOR SCIENCE. 2006. Indicators of sustainability: Reliable tools for decision making. UNESCO-SCOPE: Paris. Diponível em: <http://unesdoc.unesco.org/images/ 0015/001500/150005e.pdf>. Acesso em: 01 jan. 08.

UNWTO - UNITED NATIONS WORLD TOURISM ORGANIZATION. 2004a. Indicadores de desarrollo sostenible para los destinos turísticos: Guia práctica. UNWTO: Madrid.

. 2004b. Making tourism work for small island developing states. UNWTO: Madrid.

VAN BELLEN, Hans. 2005. Indicadores de sustentabilidade: Uma análise comparativa. Editora FGV: Rio de Janeiro.

VEIGA, José Eli. 2005. Desenvolvimento Sustentável. Rio de Janeiro: Garamond.

VICENTE, Gustavo; PARTIDÁRIO, Maria Rosário. 2006. SEA: Enhancing communication for better environmental decisions. Environmental Impact Assessment Review, v.26, n.8, p.696-706.

YUKSEL, Fisun; BRAMWELL, Bill; YUKSEL, Atila. 1999. Stakeholder interviews and tourism planning at Pamukkale, Turkey. Tourism Management, v.20, n.3, p.351-360.

\section{Recebido em: 04/02/2009}

Aprovado em: 03/09/2009 